\title{
Dewetting-mediated pattern formation inside the coffee ring
}

\author{
Weibin Li, Ding Lan," and Yuren Wang ${ }^{\dagger}$ \\ National Microgravity Laboratory, Institute of Mechanics, Chinese Academy of Sciences, 100190 Beijing, China \\ and School of Engineering Sciences, University of Chinese Academy of Sciences, 100049 Beijing, China
}

(Received 30 July 2016; revised manuscript received 24 November 2016; published 14 April 2017)

\begin{abstract}
The rearrangement of particles in the final stage of droplet evaporation has been investigated by utilizing differential interference contrast microscopy and the formation mechanism of a network pattern inside a coffee ring has been revealed. A tailored substrate with a circular hydrophilic domain is prepared to obtain thin liquid film containing monolayer particles. Real-time bottom-view images show that the evolution of a dry patch could be divided into three stages: rupture initiation, dry patch expansion, and drying of the residual liquid. A growing number of dry patches will repeat these stages to form the network patterns inside the ringlike stain. It can be shown that the suction effect promotes the rupture of the liquid film and the formation of the dry patch. The particle-assembling process is totally controlled by the liquid film dewetting and dominated by the surface tension of the liquid film, which eventually determine the ultimate deposition patterns.
\end{abstract}

DOI: 10.1103/PhysRevE.95.042607

\section{INTRODUCTION}

The evaporation of a sessile colloidal droplet occurs commonly in nature and the evaporation process involves multiple physical phenomenon including pinning effects [1,2], convection flow [1,2], dewetting [3], and capillary attraction $[4,5]$. These physical effects influence a wide range of applications such as photonic crystal formation [6], nanomaterial assembly [7], injection printing [8], and biotechnology [9,10]. As far as applications are concerned, the most important thing is to know how the deposition morphologies form and then how to control the deposition patterns. For a colloidal droplet with a contact line that is pinned at a small contact angle, the majority of solutes are carried to the edge by an outward capillary flow as occurs with evaporation, eventually forming the familiar coffee-ring pattern [11]. The coffee-ring effect can be effectively reversed by the presence of a recirculation flow during evaporation [12]. This Marangoni recirculating flow may be generated by a surfactant concentration gradient [13] or temperature gradient [14] along the droplet surface. The factors related to the direction of Marangoni flow, such as the contact angle [15], the substrate thermal conductivity [16], or the presence of impurities [17], could be used to manipulate deposition patterns $[18,19]$. In most cases, the patterns formation will be influenced by competition and cooperation between the outward capillary flow and Marangoni flow [20].

Previous works might have created an impression that the final pattern morphologies are entirely determined by various microflows inside the droplet $[2,21]$. However, in the last stage of evaporation, the pinned droplet will evolve into a thin liquid film, which spontaneously undergoes dewetting and promotes particles inside the coffee-ring redistribution to form the resulting patterns.

Recently, significant progress has been made in understanding the dewetting process of liquid film [22]. In the absence of particles, two different film rupture mechanisms have been identified that occur in films of nanoscale thickness: nucleation

\footnotetext{
*landing@imech.ac.cn

†yurenwang@imech.ac.cn
}

dewetting and spinodal dewetting [23-25]. The formation of characteristic patterns, such as annular ringlike structures [26] and dendritic structure [27] by dewetting of thin film, has been analyzed in liquid films that contain particles. In previous studies, the focus was on the dewetting of ultrathin films with or without nanoparticles, which provided a rapid bottom-up approach to create textures on a surface $[28,29]$. Compared with nanoscale liquid film, microsized film is more stable and may more easily allow the assembly of particles into ordered structures, especially a close-packed hexagonal structure. However, the dewetting process of microsized film containing microspheres still remains unclear, though it is involved in the last stage of evaporation of most colloidal droplets. Our goal is trying to provide a fundamental understanding for the interplay of liquid film dewetting and particles assembling in this final stage of the drying colloidal droplet and illustrate the formation mechanism of patterns inside the coffee ring.

In this paper we report a network pattern inside the ringlike stains that form in the final evaporation stage of colloidal droplets. We investigate the dynamic process of liquid film dewetting and microspheres assembling by utilizing differential interference contrast (DIC) microscopy and image analysis. It indicates that an uneven distribution of particles results in the rupture of liquid film and the pattern formation is controlled by the liquid film dewetting and driven by the surface tension of the liquid film.

\section{EXPERIMENTAL METHODS}

A tailored substrate is designed, as shown in Fig. 1(a). The white part is the superhydrophobic coating (SY-SupercoatSHOS150 from Shunyetech) and the black part is the circular hydrophilic quartz glass. The scanning electron microscopy (SEM) image of the cross section of the substrate shows that the thickness of the coating is nearly $60 \mu \mathrm{m}$ [Fig. 1(b)]. Such a tailored substrate is expected to confine a large colloidal droplet in the hydrophilic domain of the substrate [Fig. 1(c)], which can allow droplet evaporation in the constant contact radius mode [30] and obtain a thin liquid film containing microspheres. 


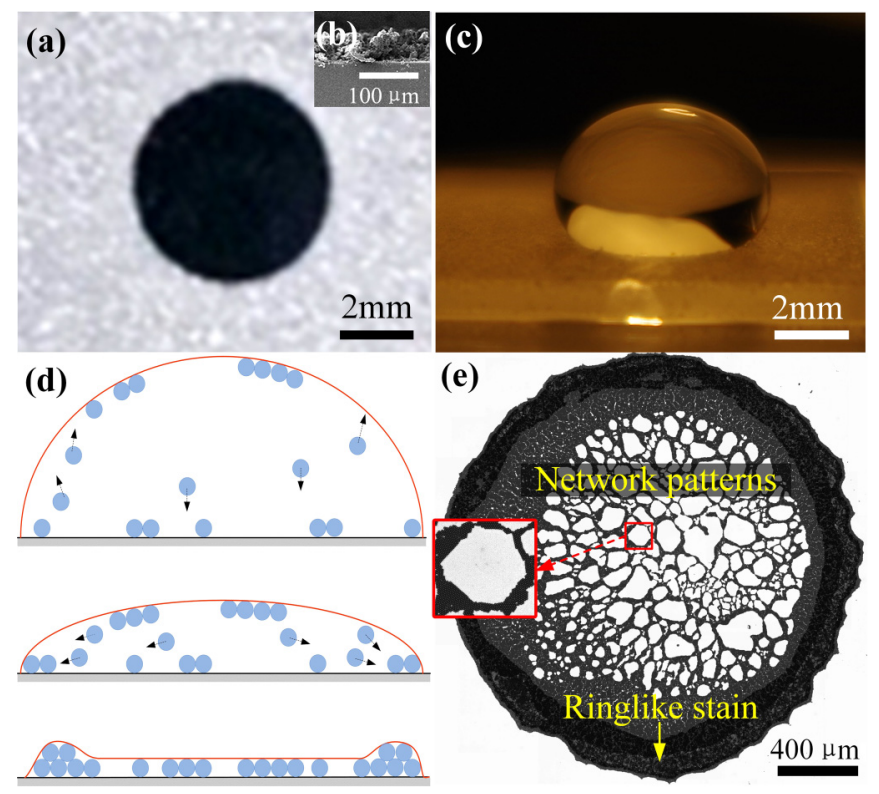

FIG. 1. (a) The tailored substrate is comprised of a circular hydrophilic domain surrounded by superhydrophobic coating. The white part is the hydrophobic domain while the black part is the hydrophilic domain. (b) The inset shows SEM image of the cross section of the substrate. (c) A large droplet could be confined in the hydrophilic domain of the substrate. (d) As with evaporation of the colloidal droplet, the self-assembly process of particles is controlled by three kinds of dynamic behavior: Marangoni flow, outward capillary flow, and capillary forces. (e) The network pattern formed inside the ringlike stain, the dry patch in the red rectangle, is a typical case that will be thoroughly analyzed.

The polystyrene (PS) colloidal microspheres, dispersed in superpure water with a mean diameter of $3.03 \mu \mathrm{m}$, are purchased from Duke Scientific Corporation (5200A). The original mass concentration is $10 \mathrm{wt} . / \mathrm{wt} . \%$ and its nominal density is $1.05 \mathrm{~g} / \mathrm{cm}^{3}$. The monodisperse PS colloidal solution used in the experiment are diluted to $0.15 \mathrm{wt} . / \mathrm{wt}$. \% with highpurity water. Different volumes of diluted colloidal solution are injected into the hydrophilic domain of the substrate after ultrasonic agitation.

To understand the dynamics of film rupture and particle migration, the evaporation process of the colloidal solution is observed and recorded simultaneously by utilizing a microscope (Nikon Ti) equipped with a high-speed digital camera. The substrate is placed on a motorized stage, which is able to move in three directions. It is used for accurately adjusting the focal plane to capture a two-dimensional (2D) flow field parallel to the substrate plane. The microscope can be used in DIC mode, which enhances the image contrast in unstained transparent samples and can generate an image showing the characteristic appearance of three dimensions. This allows the observation of the rupture and dewetting process of liquid film on the substrate. The camera is operated at a typical frame rate of 27 frames/s, sufficient to capture the evolution of the liquid film and migration of particles. All experiments are performed at environmental temperature about $20{ }^{\circ} \mathrm{C}$ and relative humidity $20 \%$. To determine the velocity of particles and the area of dry patches from captured images, IMAGE-PRO PLUS 6.0 software is used.

\section{LIQUID FILM FORMATION}

With evaporation of the colloidal droplet, the apparent contact angle decreases with time and the liquid-solid contact area remains constant [Fig. 1(d)]. The rough and the nonuniform wettability of the contact line anchors the border of the droplet, which is referred to as the canthotaxis effect [31]. In addition, the particles within the droplet accumulate at the contact line during evaporation, which could change the roughness and the wettability of the contact line and result in the self-pinning effect [32]. The droplet evaporation experiences three distinct processes. At the initial evaporation stage, the contact angle is still large enough to maintain strong recirculation flow. Two counterrotating vortex pairs could be observed in the droplet (see movies S1 and S2 in the Supplemental Material [33]), like Marangoni flow observed at the first stage in an evaporating sessile droplet by means of microparticle image velocimetry technique [34]. The irregular periphery of the hydrophilic region or the nonuniform distribution of particles in the droplet is presumably the reason to break the axial symmetry of the flow field. At this stage, colloidal particles are taken to the liquid-air interface and consequently start to clump under the force of Marangoni flow. At the second stage, the contact angle decreases and the recirculation flow is weakened with the evaporation of the droplet. In contrast, the flow pattern is mainly driven by the outward capillary flow. At this stage, the particles are pushed to the boundary of the droplet and form the ringlike stain. Due to the force of gravity, particle sedimentation can also be observed inside the ring [35]. At the third and final stage, the droplet volume decreases dramatically, although the contact line is still pinned at the periphery of the hydrophilic area. Therefore, the height of the droplet obviously decreases with the evaporation. Eventually, a thin liquid film with a thickness approximately equal to the particle size can be obtained. It should be noted that it is this third stage of the liquid film dewetting and the particle migration that we are mainly concerned with in the paper. A more detail description will be given in the following.

\section{RESULTS}

Experiments were performed on a tailored substrate with hydrophilic circular region about $4 \mathrm{~mm}$ in diameter. A colloidal droplet with a volume of $20 \mu \mathrm{L}$ was injected on the hydrophilic region. In the final seconds of evaporation, a liquid film containing monolayer particles forms inside the ringlike stain. Particles are confined on the substrate under the pressure of the liquid-air interface of the film. At the moment of the 4611th $\mathrm{s}$ in the whole evaporation process, all the particles stop moving, as shown in Fig. 2(a). This moment when particles remain stationary is defined as $0 \mathrm{~s}$. As evaporation continues, the liquid film becomes unstable and can easily be ruptured due to the effect of particles. The process of liquid film dewetting and particles migration was captured and illustrated in five typical pictures, as shown in Figs. 2(b)-2(f). When $t=0.22 \mathrm{~s}$, the liquid film initially ruptures and the first dry patch starts to form [Fig. 2(b)]. Driven by the boundary of 

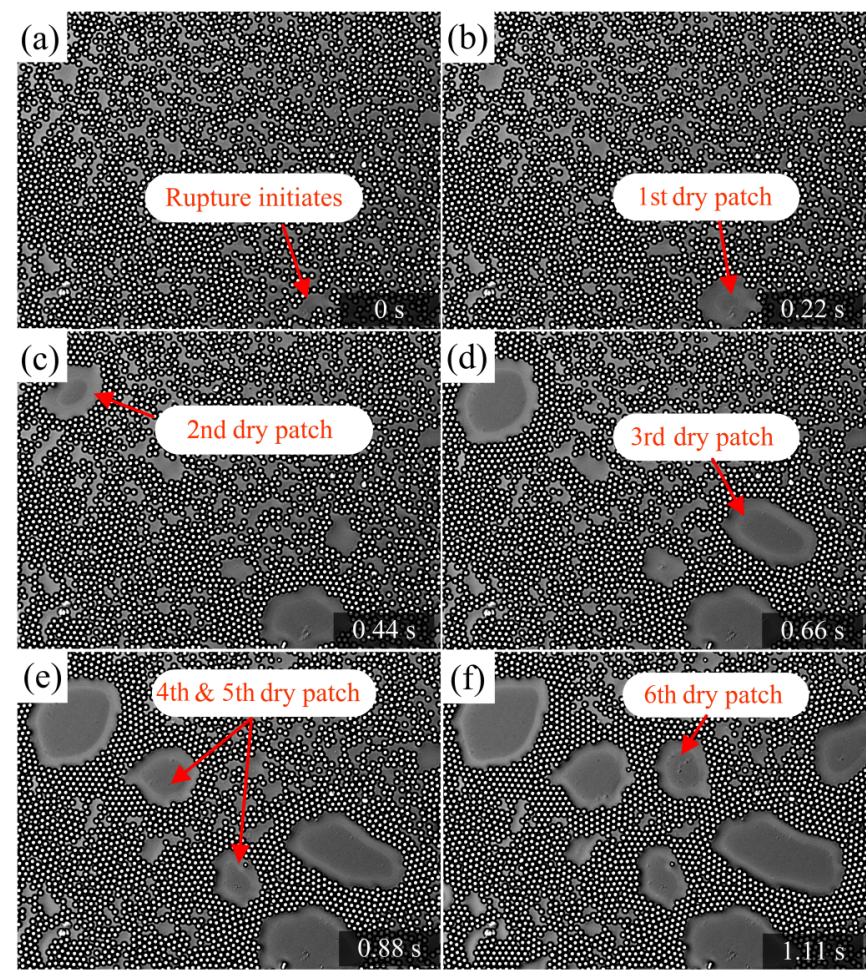

$30 \mu \mathrm{m}$

FIG. 2. Sequence of optical micrographs showing the onset and evolution of the rupture of the liquid film and aggregation of particles in the final stage of a drying droplet. (a) Rupture initiation at the particle-poor region of the substrate, (b) formation and expansion of the first dry patch, and (c)-(f) an increasing number of dry patches appear in the same way as the first one.

the dry patch, the particles around the dry patch aggregate. Along with the expansion of the first dry patch, new rupture occurs successively in other regions. A growing number of dry patches repeat the formation and expansion processes of the first one and eventually form network patterns, as shown in Figs. 2(c)-2(f). The size and distribution of these dry patches depend on the distribution of the particle density at $t=0 \mathrm{~s}$, which is further affected by many factors, such as the initial concentration, droplet volume, liquid-solid contact area, and evaporation rate. It is worth mentioning that the outward capillary flow and Marangoni flow can significantly alter the network patterns inside the ringlike stain.

To observe the formation of a general dry patch inside the ringlike stain, a colloidal drop with a volume of $5 \mu \mathrm{L}$ is injected onto the hydrophilic domain of $2.3 \mathrm{~mm}$ in diameter. The moment of the 1595th $\mathrm{s}$ in the evaporation of the droplet is redefined as $t=0 \mathrm{~s}$, as shown in Fig. 3(a1). Figures 3(a1)-3(a8) show the formation and evolution of a typical dry patch located near the center of the ringlike stain. It is about $1.15 \mathrm{~mm}$ to the periphery of the ring, as shown in Fig. 1(e). Although the expansion of the dry patch and the aggregation of particles occur almost simultaneously, they are two different physical processes. In order to clarify the coupling effect between the liquid film and particles, the dry patch, bounded by the three-phase contact line (the bright ring), is marked $S_{1}$; the particle-free region, bounded by the connecting line of the center of particles adjacent to the boundary of $S_{1}$, is labeled $S_{2}$, as illustrated in Fig. 3(a6). Two curves are plotted as shown in Fig. 3(b): The red and blue lines reflect the area of the dry patch and the area of the particle-free region, respectively, and the sizes of these areas change with time. The trend of these two curves indicates that this final drying process can be subdivided into three stages, as illustrated in the schematic diagram in Fig. 3(c). In stage I, when $t=0 \mathrm{~s}$, all the particles are confined between the liquid-air interface and the substrate and remain stationary. As the liquid evaporates, the local liquid film at the particle-free region is thinning with time and forms a concave surface among particles. Then particles would be pushed due to the deformation of the liquid-air interface, as shown in Fig. 3(a2). In this stage, the dry patch does not yet appear, but the particle-free region $\left(S_{2}\right)$ starts to expand. In stage II, when the lowest concave surface has reached the substrate, the liquid film is disrupted by the creation of the dry patch [Fig. 3(a3)]. It is observed that the dry patch forms in the particle-poor region. By encompassing particles along its rim, the dry patch expands along the radial direction in less than 1s [Figs. 3(a3)-3(a6)]. The two curves in Fig. 3(b) show that the expansions of the dry patch and the particle-free region are synchronous, which indicates that the particle aggregation process is driven by the liquid film dewetting in this stage. In stage III, the particle-free region remained unchanged as all of the closely packed particles are compacted and deposited on the substrate [Fig. 3(b)]. The dry patch continues to expand until the wetting film covering the patterns eventually dries, as shown in Figs. 3(a7) and 3(a8). The formation and evolution of this dry patch is a typical case; all other dry patches at different locations show the same behavior. Many of these dry patches and close-packed structures constitute the network pattern inside the ringlike stain (see movie S3 in the Supplemental Material [33]).

\section{DISCUSSION}

The uneven distribution of particles on the substrate is critical for the dry patch formation. As occurs during evaporation, particles will be partially immersed in the wetting liquid film. The deformation of the liquid-air interface gives rise to interparticle capillary forces. Because particles are randomly distributed on the substrate, there are differences in the spacing and liquid volumes among the particles, which may lead to the inequality of capillary forces; this may be the underlying factor driving dry patch formation [36]. The increased evaporation decreases the level of the liquid between the particles and increases the curvature of the menisci; every meniscus acts like a low-pressure pump trying to suck liquid from other places [37]. Because of the uneven sizes of pores, the menisci in large pores can produce larger pressure, which pushes liquid to menisci in small pores, as shown in Fig. 4(a).

The experimental results indicate that the dry patch initially forms in the particle-poor region. To qualitatively analyze the formation of the dry patch, a geometrical model is constructed as illustrated in Fig. 4(a). The liquid meniscus is simplified as a spherical surface; therefore, one obtains the geometric relationship $\left(\frac{D}{2}+\rho\right) \sin \phi=\frac{L}{2}$, where $D$ is the diameter of the colloidal particle, $\rho$ is the radius of curvature of the liquid meniscus, $\phi$ is the meniscus slope angle corresponding to an 

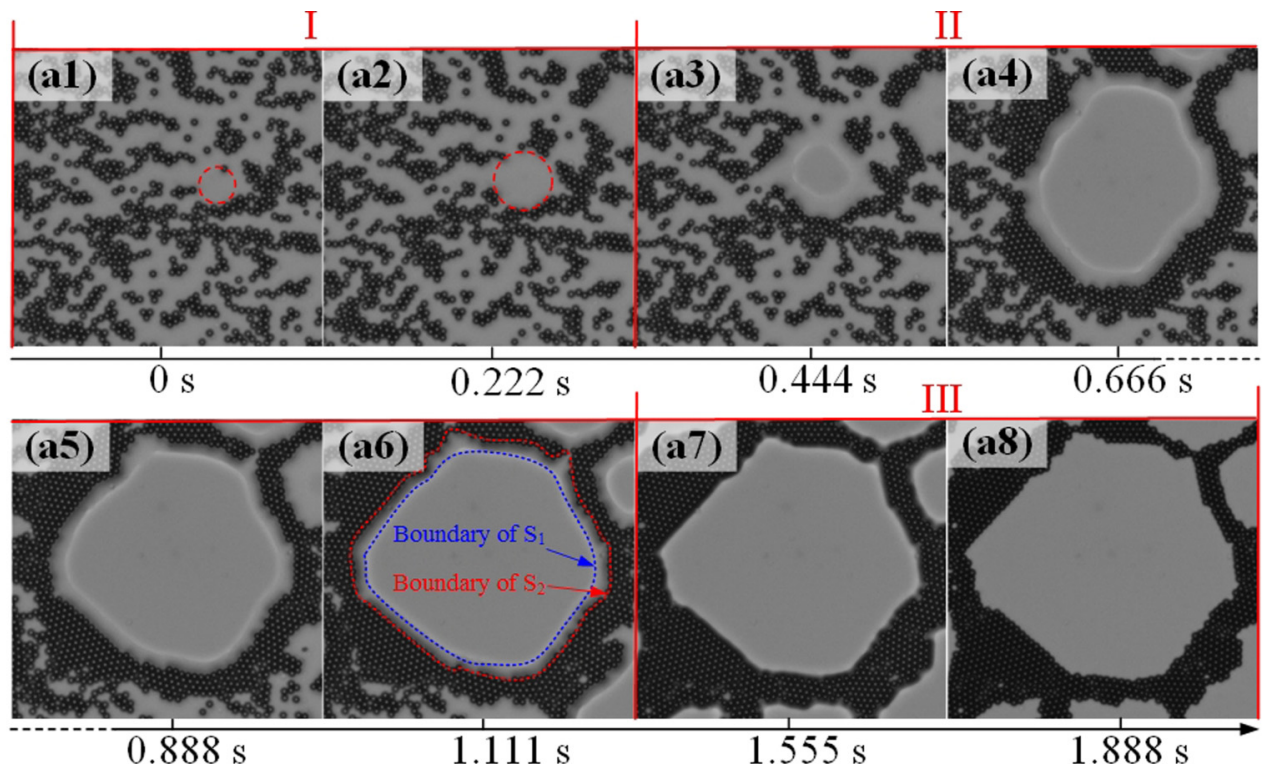

(b)

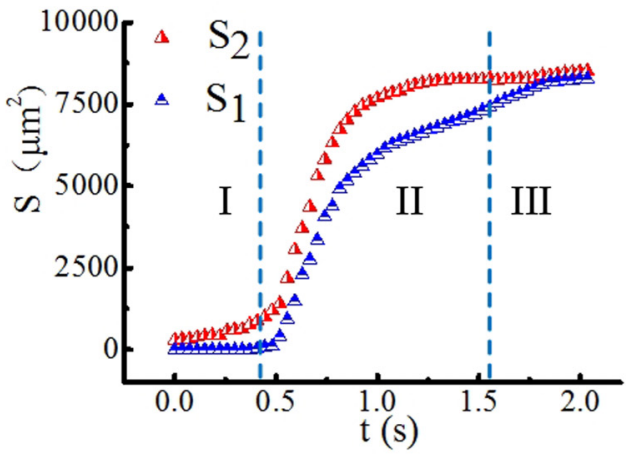

(c)

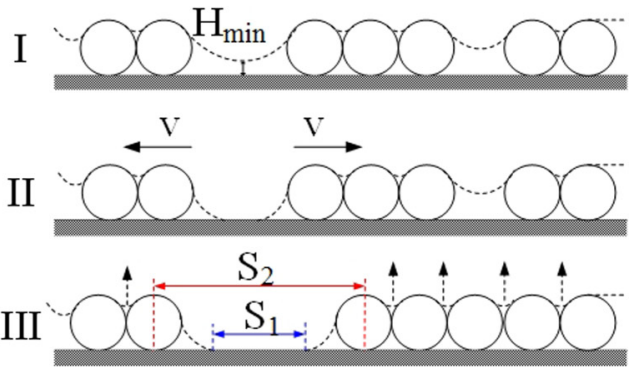

FIG. 3. (a1)-(a8) Successive stages of the formation and evolution of a typical dry patch $\left(S_{1}\right)$ and particle-free region $\left(S_{2}\right)$. The dry patch is surrounded by the three-phase contact line (boundary of $S_{1}$ ), while the particle-free region is the area surrounded by particles (boundary of $S_{2}$ ). (a1) and (a2) In stage I the particle-free region expands due to the deformation of liquid film and film rupture initiates at the particle-poor region as shown in the red circle. (a3)-(a6) In stage II the dry patch forms and expands and particles aggregate by dewetting of the liquid film. (a7)-(a8) In stage III a close-packed structure forms and the residual liquid dries (see movie S4 in the Supplemental Material [33]). (b) Plot of the measured area of the dry patch $\left(S_{1}\right)$ and the particle-free region $\left(S_{2}\right)$ versus time. (c) Schematic presentation of the formation and evolution of a dry patch.
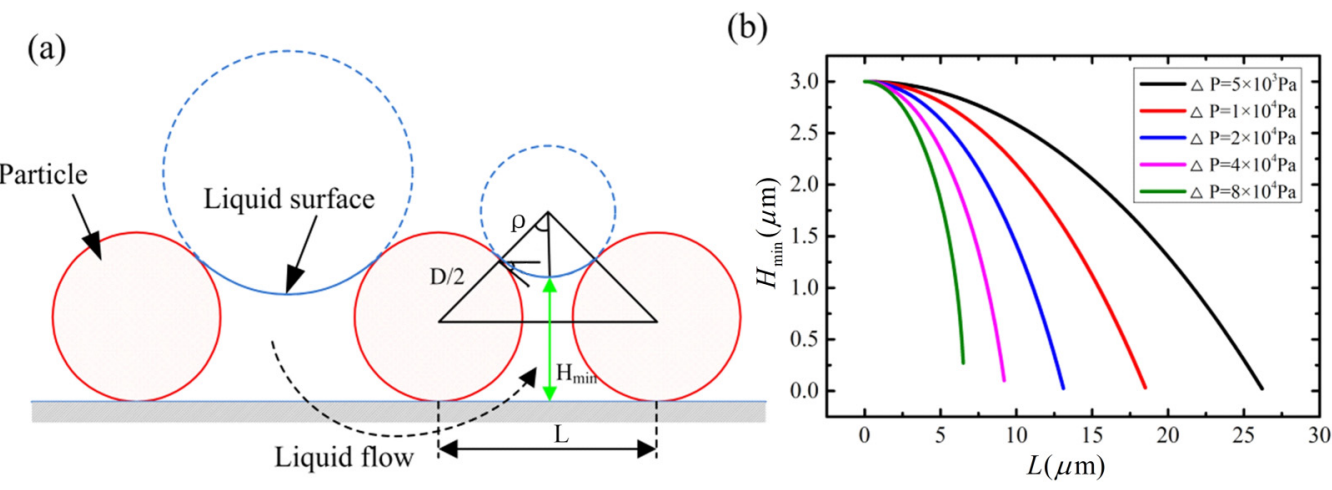

FIG. 4. (a) Schematic of the liquid flow caused by the uneven distribution of particles. The pressure in the large pore is larger than that in the small pore, which induces liquid flow from the large pore to the small pore and eventually makes the film rupture in the large pore. (b) The minimum thickness of the liquid film changes with particle spacing under a certain pressure difference. The various values of the pressure difference are estimated from the Laplace equation base of experimental data. 


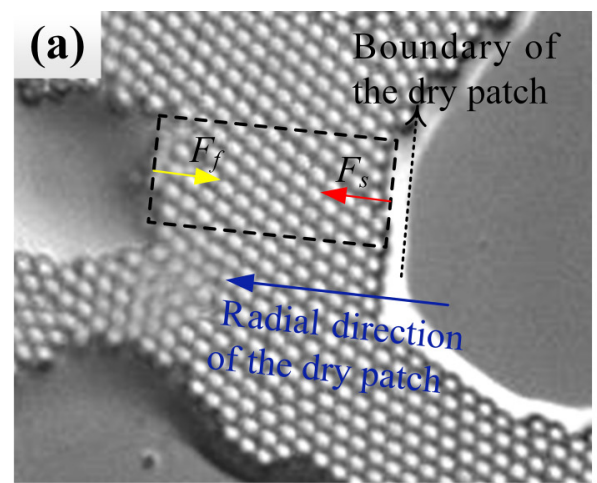

(c)
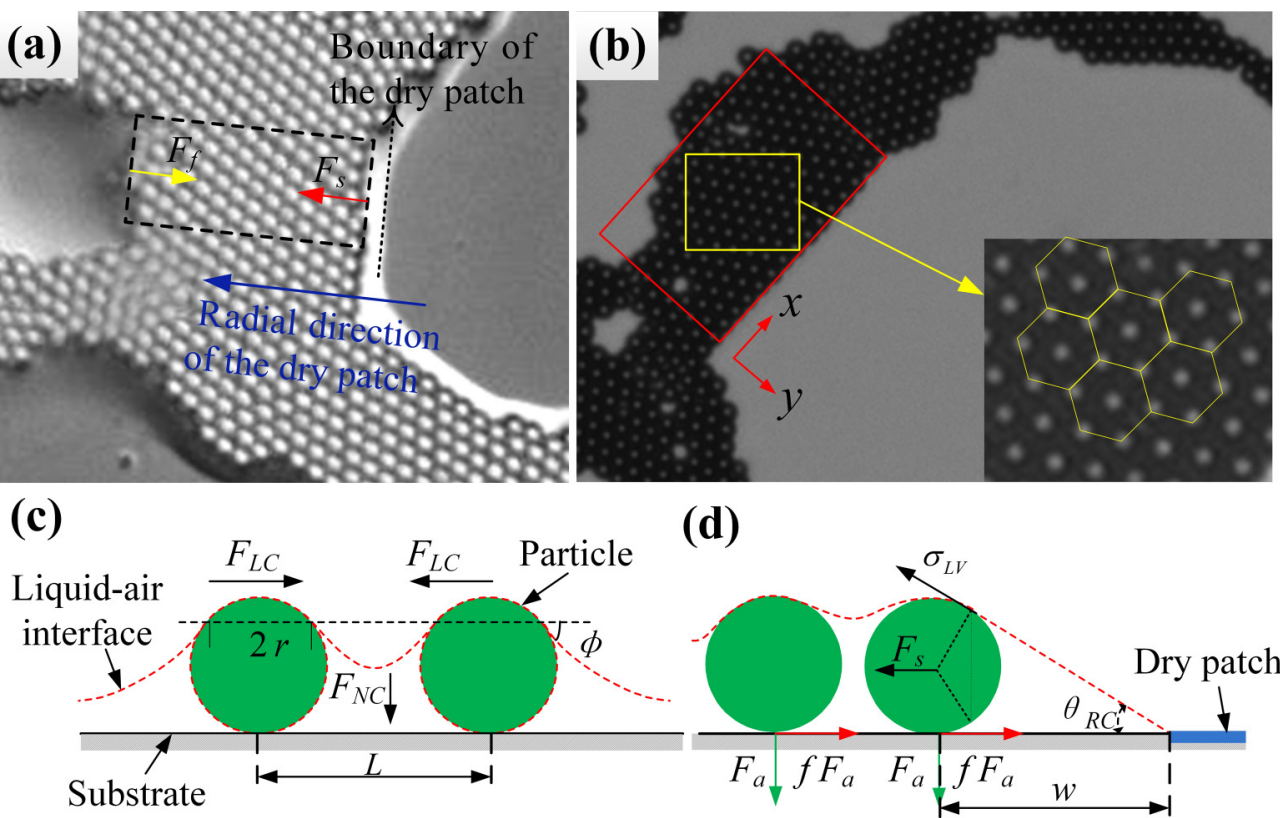

(d)

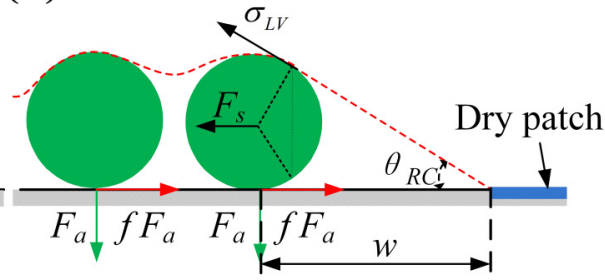

FIG. 5. Analysis diagram of the influence of the liquid film dewetting on the particles assembling. (a) Microscopic image of the particle aggregation process captured by an interference microscope. The frictional force $F_{f}$ increases to resist the surface tension force $F_{s}$ along the radial direction of the dry patch. (b) Image of the local structure of the dry patch in Fig. 3(a8). The structure in the yellow rectangular is enlarged and presents a close-packed hexagonal array. The structure in the red rectangular is chosen to estimate the equivalent number of particles $\bar{n}$ in the experiment. (c) Lateral and normal capillary forces generated by the menisci formed around two particles. (d) Schematic of forces acting on particles adjacent to the dry patch.

arbitrary cross section of the meniscus, and $L$ is the particle spacing. Then the thickness of the thinnest liquid film $H_{\text {min }}$ can be expressed as

$$
H_{\min }=\left(\frac{D}{2}+\rho\right) \cos \phi+\frac{D}{2}-\rho .
$$

As we simplified the meniscus as a spherical surface, the Young-Laplace equation can be expressed as $\Delta P=P_{0}-$ $P_{\mathrm{atm}}=\frac{2 \sigma_{L V}}{\rho}$ [38]. After substituting this into (1), we get

$$
H_{\min }=\left(\frac{D}{2}+\frac{2 \sigma_{L V}}{\Delta P}\right) \sqrt{1-\frac{L^{2}}{\left(D+\frac{4 \sigma_{L V}}{\Delta P}\right)^{2}}}+\frac{D}{2}-\frac{2 \sigma_{L V}}{\Delta P}
$$

where $\Delta P$ is the pressure difference between the inside and outside of the meniscus, the various values of the pressure difference $\Delta P$ in Fig. 4(b) can be estimated from the Laplace equation based on experimental data, $\sigma_{L V}=72 \mathrm{mN} / \mathrm{m}$ is the surface tension of water, and $D=3 \mu \mathrm{m}$ is the particle diameter. Figure 4(b) shows the variation in thickness of the thinnest film $H_{\min }$ as a function of particle spacing $L$. We observe a nonlinear decrease that becomes steeper for a larger pressure difference. If the pressure difference $\Delta P$ remains constant, the pressure generated in the particle-rich region is larger than that in the particle-poor region, which induces liquid flow from the particle-poor region to the particle-rich region, just like the suction effect caused by uneven sizes of pores [37]. Therefore, the liquid film should be thinner at the particle-poor region, and this thinner liquid film more easily ruptures. As with evaporation of the liquid, the pressure difference increases with the rise of curvature of the meniscus
[Fig. 4(b)], the suction effect is enhanced, and the rupture of the liquid film is accelerated.

In the dewetting process of the liquid film containing particles, the free liquid surface is mainly responsible for the emergence of patterning. It can be deduced that there is a complex interplay between liquid film dewetting and particle aggregation. Here the effect of the liquid film dewetting on the particles assembling is illustrated. Dewetting is closely related to the wettability of the substrate, which can be represented by Young's equation $\sigma_{S V}-\sigma_{S L}=\sigma_{L V} \cos \theta$, where $\sigma_{S V}, \sigma_{S L}$, and $\sigma_{L V}$ represent the interfacial tensions of the solid-vapor, solid-liquid, and liquid-vapor interfaces, respectively [39]. The receding contact angle (RCA) $\theta_{R}$, a preferable criterion in the wettability of the substrate, is about $22^{\circ}$ for the hydrophilic domain of the tailored substrate. The time-dependent RCA is defined as the dynamic receding contact angle (DRCA) $\theta_{R C}$, which can be expressed as the equation $\tan \theta_{R C} / 2=R / w$, as shown in Fig. 5(d). The radius $R$ of the particles is $3 \mu \mathrm{m}$ and the wetting length $w$, as a function of time, can be obtained experimentally. From this, the relationship between the DRCA and time could be obtained as shown in Fig. 6(b). From the curves of Fig. 6(a) we know that the particle migration process is completely controlled by the expansion of the dry patch. A comparison of Figs. 6(a) and 6(b) shows that when $t \leqslant 1.1 \mathrm{~s}$, the DRCA is less than the RCA. The DRCA moves towards restoration of the RCA for the release of energy in the system [40], so the dry patch expands instantly. The particles around the dry patch are pushed by the film rim to aggregate. When $t>1.1 \mathrm{~s}$, the DRCA is larger than the RCA, the local particles around the dry patch remain stationary and cannot be pushed further by the film rim. The dry patch continues to expand due to the suction effect caused by evaporation. Finally, 
(a)

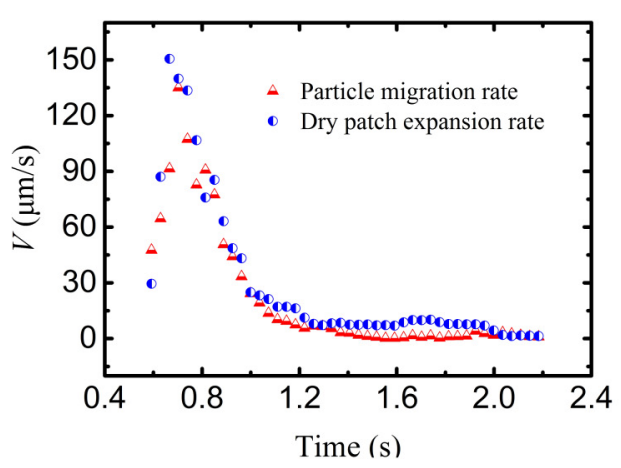

(b)

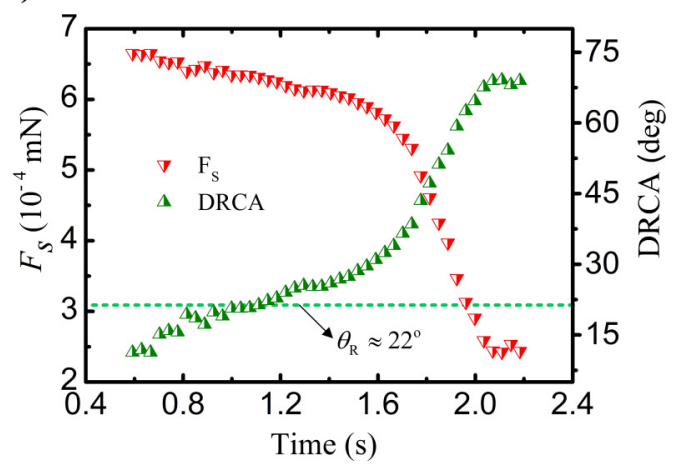

FIG. 6. (a) Calculated particle migration rate and dry patch expansion rate as a function of time. (b) Estimated surface tension force $F_{s}$ and the DRCA variance with time. The RCA of the hydrophilic domain of the tailored substrate is about $22^{\circ}$.

particles squeeze each other to form a close-packed hexagonal structure in the particle-rich region, just like the structure in the inset of the amplified yellow rectangular region in Fig. 5(b). Analysis of different dry patches inside the ringlike stain shows similar behavior. It is therefore valid to conclude that the liquid film dewetting contributes to the particles assembling in two different ways over time: restoration of the RCA and the suction effect.

During the process of particles assembling, the surface tension of the liquid film is dominant. The research of Kralchevsky and Denkov suggests that the menisci formed around two adjacent particles confined in a liquid film will generate two types of capillary forces, the lateral capillary force $F_{L C}$ and the normal capillary force $F_{N C}$, as shown in Fig. 5(c) [4]. The lateral capillary force can be simplified as [41]

$$
F_{L C}=\pi \sigma_{L V} Q^{2} / L
$$

The normal capillary force is given by

$$
F_{N C}=\pi r \sigma_{L V} \sin \phi,
$$

where $Q=r \sin \phi$ is called the capillary charge and $\mathrm{r}$ is the radial distance, as shown in Fig. 5(c). The lateral capillary force tends to attract and bring particles together and accelerates the ordering of particles. The normal capillary force increases and enhances the particle adhesion (attraction) to the substrate.

Particle motion near the dry patch is a result of the interplay between various interaction forces as depicted in Fig. 5(d). The surface tension force $F_{s}$, which is equivalent to the surface tension that the liquid-air interface exerts on the particles trapped in the rim of the film, can be expressed as [42]

$$
F_{S}=2 \pi R \sigma_{L V} \cos \theta_{R C} .
$$

The surface tension force decreases with time, as shown in Fig. 6(b). The frictional force $F_{f}$, which is caused by the adhesion between the particles and substrate, can be expressed as

$$
F_{f}=\bar{n} f F_{a},
$$

where $\bar{n}$ is the equivalent number of particles arrayed as a line along the radial direction of the dry patch, as shown in Fig. 5(a); $f$ is the friction coefficient; and $F_{a}$ is the total adhesive force, which includes the force due to gravity, the electrostatic force, the van der Waals force, and the normal capillary force. The first three forces can be neglected compared with the normal capillary force, so $F_{f}$ can be derived from Eqs. (4) and (6),

$$
F_{f}=\bar{n} f F_{N C}=\pi \bar{n} f r \sigma_{L V} \sin \phi .
$$

Because the equivalent number of particles $\bar{n}$ and the normal capillary force $F_{N C}$ increase with dry patch expansion, the frictional force $F_{f}$ increases with time until particles along the radial direction of the dry patch stop moving. In the expansion process of the particle-free region $\left(S_{2}\right)$, the surface tension force acting on the particles tends to push them away, whereas the frictional force tends to stop particles from moving, as shown in Fig. 5(a). It is obvious that $F_{f} \geqslant F_{s}$ is the necessary condition for the end of particle movement; thus the equivalent number of particles $\bar{n}$ required for particles coming to rest is then given by

$$
\bar{n} \geqslant \frac{F_{s}}{\pi f r \sigma_{L V} \sin \phi} .
$$

For the ideal 2D close-packed hexagonal structure, such as the particle arrangement in the rectangular region in Fig. 5(a), the equivalent number of particles $\bar{n}$ is approximately equal to the number of rows (the row direction is parallel to the boundary of the dry patch) of the structure.

Considering the situation of stage III around the dry patch in Fig. 3(a), when $t=1.554 \mathrm{~s}$, the particles just stop moving [Fig. 6(a)] and the surface tension force $F_{s}$ is $5.9 \times 10^{-4} \mathrm{mN}$ [Fig. 6(b)]. Using the friction coefficient $f=0.17$ [43], the radial distance $r=1.5 \mu \mathrm{m}$, and the extremum of the meniscus slope angle $\phi=90^{\circ}$, which can be calculated from Fig. 4(a), the equivalent number of particles can be estimated to be $\bar{n} \geqslant 10$. This indicates that more than 10 rows of particles could prevent expansion of the boundary of the particle-free region $\left(S_{2}\right)$. The structure inside the red rectangle in Fig. 5(b) was analyzed for its minimal impact from the adjacent dry patch, i.e., the particle aggregation in this region is mainly dominated by the dry patch at the lower right, but is hardly affected by the dry patch at the upper left (see movie S3 in the Supplemental Material [33]). It can be seen that 11 rows (along the $x$ direction) of the particle arrangement along the direction of the frictional forces (the $y$ direction) will prevent particles from moving, which is consistent with our prediction. Using Eq. (8), one can better understand the physical mechanism of 
particles aggregation and assembly; however, the mechanical behavior of particles in reality is very complex. The equivalent number of particles $\bar{n}$ may deviate from the predicted value, due to the irregular boundary of the dry patch, the uneven initial distribution of particles, or the mutual influences of the adjacent dry patches. The lateral capillary force $F_{L C}$, together with the surface tension force $F_{S}$, drives particle aggregation and assembly; the normal capillary force $F_{N C}$, along with the frictional force $F_{f}$, could prevent the movement of particles. All these forces stem from the surface tension of the liquid film. Therefore, the surface tension of the liquid film controls the formation process of network patterns inside the ringlike stain.

\section{CONCLUSION}

This work has described a network pattern inside the coffee ring. At the final seconds of evaporation, the pinned droplet evolves into a thin liquid film. The dynamic processes of liquid film dewetting and the particles assembling were investigated by utilizing DIC microscopy. Experimental results show that the evolution of a dry patch could be divided into three stages: rupture initiation, dry patch expansion, and drying of the residual liquid. A growing number of dry patches will repeat these stages to form the network patterns inside the ringlike stain. A geometrical model was set up to analyze the formation mechanism of the dry patch. It is indicated that rupture of the liquid film tends to happen at the particle-poor region, which can be attributed to the suction effect caused by an uneven distribution of particles. The time evolution of the DRCA and the particle migration rate show that the aggregation of particles is controlled by the expansion of the dry patch and the liquid film dewetting contributes to the assembly of particles in two different ways over time: restoration of the RCA and the suction effect. The analysis of the various forces acting on particles revealed that the particle assembly is dominated by the surface tension of the liquid film, which eventually determine the ultimate deposition patterns. We believe that there is a complex and strong interplay between liquid film dewetting and particle aggregation. The nonuniform distribution of particles promotes the rupture of the liquid film and the liquid film in turn dominates the particle assembly process.

\section{ACKNOWLEDGMENTS}

We thank R. Liu for helpful discussions on the theory of dewetting of liquid film and reading the manuscript. We gratefully acknowledge financial support from National Natural Science Foundation of China (Grant No. 11472275), Strategic Priority Research Program on Space Science, the Chinese Academy of Sciences (A) (Grants No. XDA04020202 and No. XDA04020406), and the Strategic Priority Research Program of the Chinese Academy of Sciences (Grant No. XDB22040301).
[1] W. Han and Z. Lin, Angew. Chem. Int. Ed. 51, 1534 (2012).

[2] R. G. Larson, AIChE J. 60, 1538 (2014).

[3] A. Stannard, J. Phys.: Condens. Matter 23, 083001 (2011).

[4] P. A. Kralchevsky and N. D. Denkov, Curr. Opin. Colloid Interface Sci. 6, 383 (2001).

[5] P. J. Yunker, T. Still, M. A. Lohr, and A.G. Yodh, Nature (London) 476, 308 (2011).

[6] D. J. Norris, E. G. Arlinghaus, L. Meng, R. Heiny, and L. Scriven, Adv. Mater. 16, 1393 (2004).

[7] Y. Xie et al., Langmuir 29, 6232 (2013).

[8] A. Shimoni, S. Azoubel, and S. Magdassi, Nanoscale 6, 11084 (2014).

[9] T.-S. Wong, T.-H. Chen, X. Shen, and C.-M. Ho, Anal. Chem. 83, 1871 (2011).

[10] A. Askounis, Y. Takata, K. Sefiane, V. Koutsos, and M. E. Shanahan, Langmuir 32, 4361 (2016).

[11] R. D. Deegan, O. Bakajin, T. F. Dupont, G. Huber, S. R. Nagel, and T. A. Witten, Nature (London) 389, 827 (1997).

[12] H. Hu and R. G. Larson, J. Phys. Chem. B 110, 7090 (2006).

[13] T. Still, P. J. Yunker, and A. G. Yodh, Langmuir 28, 4984 (2012).

[14] H. Hu and R. G. Larson, Langmuir 21, 3972 (2005).

[15] X. Xu, J. Luo, and D. Guo, Langmuir 26, 1918 (2010).

[16] W. D. Ristenpart, P. G. Kim, C. Domingues, J. Wan, and H. A. Stone, Phys. Rev. Lett. 99, 234502 (2007).

[17] A. Marin, R. Liepelt, M. Rossi, and C. J. Kähler, Soft Matter 12, 1593 (2016).

[18] D. J. Harris and J. A. Lewis, Langmuir 24, 3681 (2008).
[19] V. X. Nguyen and K. J. Stebe, Phys. Rev. Lett. 88, 164501 (2002).

[20] B. M. Weon and J. H. Je, Phys. Rev. E 87, 013003 (2013).

[21] A. K. Thokchom, A. Gupta, P. J. Jaijus, and A. Singh, Int. J. Heat Mass Transfer 68, 67 (2014).

[22] A. Nikolov and D. Wasan, Adv. Colloid Interface Sci. 206, 207 (2014).

[23] G. Reiter, Phys. Rev. Lett. 68, 75 (1992).

[24] M. Elbaum and S. G. Lipson, Phys. Rev. Lett. 72, 3562 (1994).

[25] U. Thiele, M. Mertig, and W. Pompe, Phys. Rev. Lett. 80, 2869 (1998).

[26] P. C. Ohara and W. M. Gelbart, Langmuir 14, 3418 (1998).

[27] G. F. Harrington, J. M. Campbell, and H. K. Christenson, Cryst. Growth Design 13, 5062 (2013).

[28] C. A. Rezende, L.-T. Lee, and F. Galembeck, Langmuir 23, 2824 (2007).

[29] D. Gentili, G. Foschi, F. Valle, M. Cavallini, and F. Biscarini, Chem. Soc. Rev. 41, 4430 (2012).

[30] R. Picknett and R. Bexon, J. Colloid Interface Sci. 61, 336 (1977).

[31] T. Ondarçuhu and M. Veyssié, J. Phys. (France) II 1, 75 (1991).

[32] B. M. Weon and J. H. Je, Phys. Rev. Lett. 110, 028303 (2013).

[33] See Supplemental Material at http://link.aps.org/supplemental/ 10.1103/PhysRevE.95.042607 for the experimental movies.

[34] A. Yagodnitsyna, A. Bilsky, M. Roudgar, J. D. Coninck, and O. Kabov, MATEC Web Conf. 84, 00042 (2016).

[35] N. R. Devlin, K. Loehr, and M. T. Harris, AIChE J. 62, 947 (2016). 
[36] Z. Zhou, Q. Li, and X. Zhao, Langmuir 22, 3692 (2006).

[37] L. Xu, S. Davies, A. B. Schofield, and D. A. Weitz, Phys. Rev. Lett. 101, 094502 (2008).

[38] T. Stifter, O. Marti, and B. Bhushan, Phys. Rev. B 62, 13667 (2000).

[39] T. Young, Philos. Trans. R. Soc. London 95, 65 (1805).
[40] C. Redon, F. Brochard-Wyart, and F. Rondelez, Phys. Rev. Lett. 66, 715 (1991).

[41] A. D. Nikolov and D. T. Wasan, Ind. Eng. Chem. Res. 48, 2320 (2008).

[42] J.-Y. Jung, Y. W. Kim, J. Y. Yoo, J. Koo, and Y. T. Kang, Anal. Chem. 82, 784 (2010).

[43] V. H. Chhasatia and Y. Sun, Soft Matter 7, 10135 (2011). 